\title{
Modelling and simulation for optimization of a Jatropha oil extraction process line for operations management using Witness
}

\author{
dominic Onimowo ${ }^{1}$ \\ ${ }^{1}$ Colchester Institute
}

May 5, 2020

\begin{abstract}
Inefficiency in operations management cause wastage of resources and funds. This paper will define and properly describe the supply chain problem, build a representative simulation model, validate it using witness and then find a solution to the problem. The process line was optimised, and the efficiency increased to about $95 \%$.
\end{abstract}

\section{Keywords: Jatropha, Witness, process line optimization, operations management 1.0 INTRODUCTION}

A supply chain can be described as all the encompassing processes involved in fulfilling a customer's needs (Chen et al., 2019). The Jatropha plant is a non-edible shrub that grows in the tropical and sub-tropical regions. It has a very high resistance to aridity hence it can be grown in dessert lands. It is about thirty percent oil per seed content, eighty percent per seed content when extracted and can be used as a source of biodiesel when processed (Keith, 2000). A hectare of Jatropha plantation can yield about six hundred litters of oil. The process line to be modelled concentrates on the plantation to oil extraction stage.

\subsection{PROCESS OVERVIEW}

2.1 system data collection

This is the stage where all data collection is carried out. The capacity of the various machinery involved in the process line are determined. This process is very necessary to avoid the process collapsing. First adequately defining the aim of the process, which was done above, and the end and result got the integrated definition planning flow chart below. The process was then designed using witness and any superfluous operations, which cost money or impeded operations, were removed.

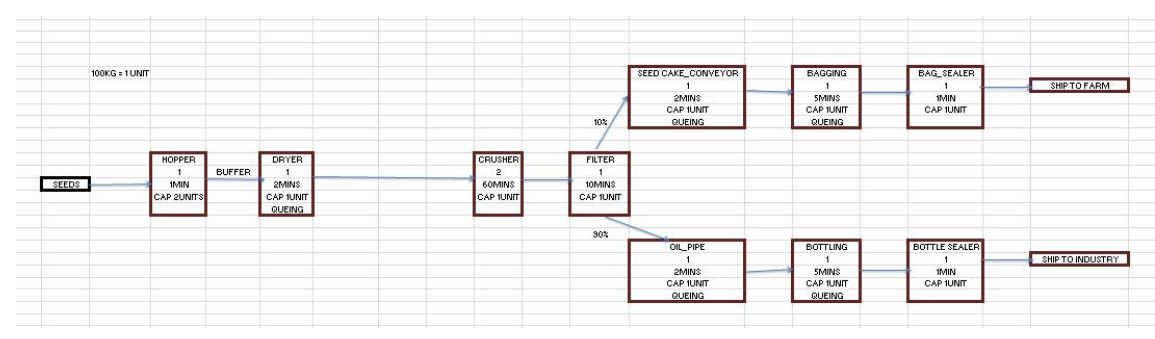

Figure 1: integrated definition planning flow chart of seed to product shipment

The diagram above shows an integrated definition planning flow chart of the process line from raw material to product distribution arranged in layers. The first layer shows the nomenclature of the machinery needed 
at that point, the second layer gives the number of the machines present at that point and the third gives the time $t$ will take the machine to carry out the task given while for those with a fourth layer like the conveyors it shows their queuing capacity. This has helped us know the full operating capacity of the production line and the bottleneck of the process line. The seeds are measured in kilograms but for ease of operations they are equated to units that is one kilogram is equal to one unit. The seeds, which are the raw material represented as part in witness simulation move to the hopper with a capacity of $200 \mathrm{~kg}$ that is two units holds them for one minute (sixty seconds).

The dryer removes moisture from the seeds and has a capacity of $100 \mathrm{~kg}$ (one unit) has a cycle time of 2minutes (one hundred and twenty seconds). The next machine is the crusher where the oil is extracted by applying pressure on the seeds for one-hour (three thousand six hundred seconds) with a 100kg (one unit) capacity after which the crushed seed cake and oil are transferred to the filter. The filter works for ten minutes (six hundred seconds), takes one hundred kilograms (one unit), separates the seed cake from the oil and this is currently at sixty percent oil and forty percent seed cake, which is not so efficient. At this point the process line splits into two, Cake conveyor collects the seed cake and transports this for two minutes (one hundred and twenty seconds) while oil pipe collects the extracted oil also for two minutes (one hundred and twenty seconds).

The seed cake is bagged at $100 \mathrm{~kg}$ per bag, which takes five minutes (three hundred seconds) and transferred to the bag sealer, which takes only a minute (sixty seconds). This seals the bags and from there they are shipped to the farms for use as fertilizer. The extracted oil from the oil pipe is transferred to the bottle machine, which also bottles at $100 \mathrm{~kg}$ per bottle taking five minutes (three hundred seconds) and the bottle is sent to the bottle sealer, which seals then ships to the industry for further processing. The total process time is about one hundred and forty-one thousand six hundred seconds.

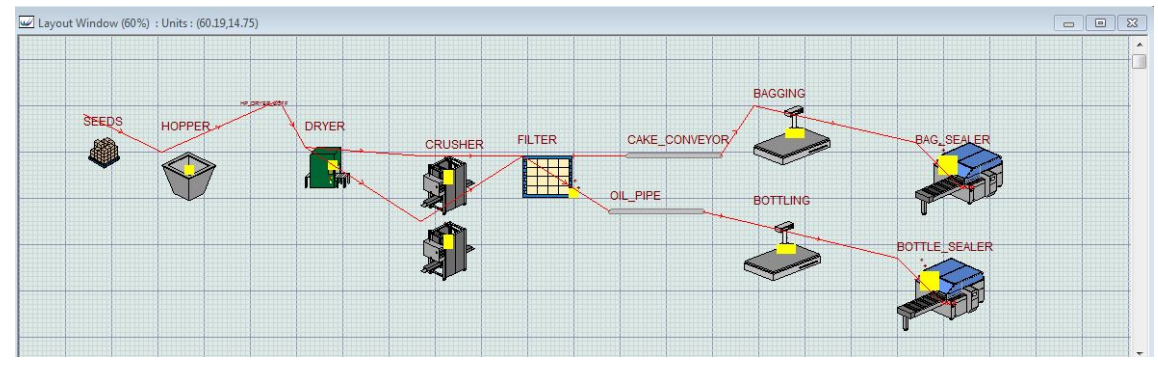

Figure 2: witness model of process

\subsection{PROCESS BREAKDOWN}

\section{Seeds}

This is the raw material needed for the process it is a passive material hence it can be pulled anytime, as it is always available. They are pulled by the hopper, which stores them temporarily before drying.

\section{Hopper}

This is a temporary storage for the seeds before drying. The hopper holds about $200 \mathrm{~kg}$ of seed equivalent to 2 units and has a cycle time of about sixty seconds.

\section{Dryer}

The dryer is used to reduce the moisture content of the seeds to the required amount.it works like a conveyor as the seeds are passed through it a wave of heat is sent through which dries or reduces the moisture from the seed. The dryer capacity is one unit and it operates for one hundred and twenty seconds. 


\section{Buffer}

This is a necessary measure for a region where there is a possibility of slow movement of part accumulation. The buffer was implemented since the carrying capacity of the dryer is less than that of the hopper hence there will be delays in transmission so the part that cannot be dried immediately which is about $100 \mathrm{~kg}$ is stored temporarily in the buffer region.

Crusher

This is the bottleneck of the whole extraction process. It has a cycle time of about three thousand six hundred seconds and has a capacity of one unit. The crusher makes use of pressure to extract oil from the seeds.

Filter

This part of the chain is used to separate the cake from the oil. It takes about six hundred seconds and has a capacity of $100 \mathrm{~kg}$. The product from the filter is divided using the percentage rule according to the efficiency, sixty percent of the filtered produced which is oil is taking to the oil pipe while the remaining forty percent which is seed cake is taken to the cake conveyor.

Cake conveyor

After separation, the current efficiency system makes provision for forty percent of the crushed produce which is seed cake to flow into the cake conveyor, which accommodates one unit per length or as adapted to this case per kilogram.

Oil pipe

This is also a conveyor and from the efficiency system, it is allowed sixty percent of the produce, which is oil. It transfers this oil to the bottling machine.

\section{Bagging}

This machine has a cycle time of three hundred seconds and a one hundred per kilogram capacity. It is used to package the seed cake.

\section{Bottling}

This machine is used to bottle the oil produced and has a cycle time of three hundred seconds and a capacity of $100 \mathrm{~kg}$.

Bag sealer

This is used to seal up the already packaged seed cakes. It takes one minute.

Bottle sealer

The machine is used to seal up the bottled oil in a one-minute cycle.

When the process is completed, the sealed cake bags are shipped of to the farm for use as fertilizer while the sealed oil bottles are shipped of to the industry for further processing. 


\subsection{PROCESS SETBACK}

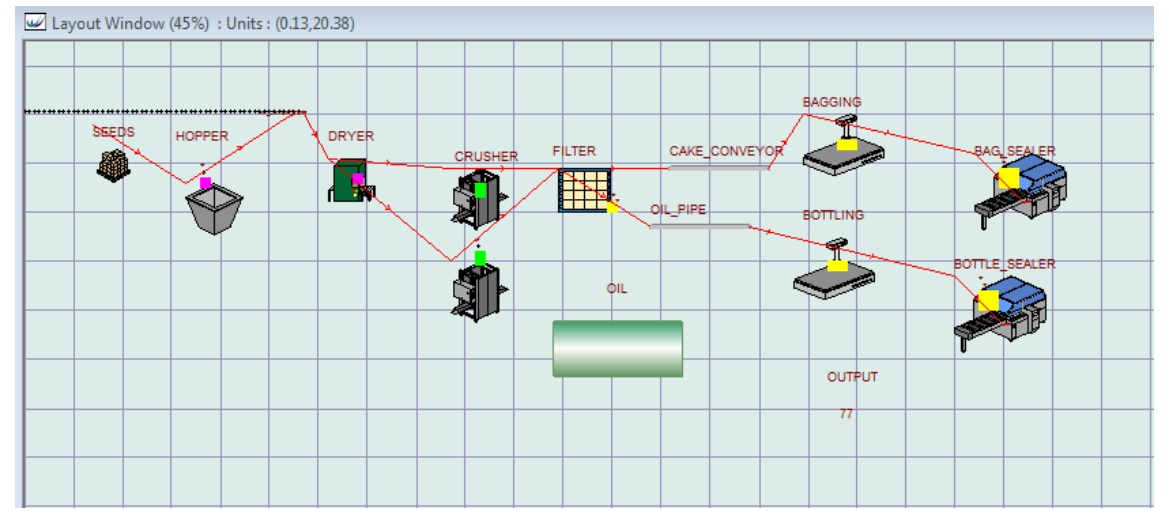

Figure 3: Run 1

The process as designed has been in use for a very long time and is very ineffective as it is. Figure 3 shows the first run of the simulation and as shown the output is one less than the real output. Knowledge gained from the study of modelling and simulation for operations management and research into this field we have learnt that the process cycle time can be much quicker and more efficient.

\begin{tabular}{|c|c|c|c|c|c|c|c|c|c|}
\hline$\square$ WITNESS & & & & & & & & & \\
\hline \multicolumn{10}{|c|}{ Part Statistics Report by On Shift Time } \\
\hline Name & No. Entered & No. Shippe & No. Scrapp & No. Assem & No. Rejecte & W.I.P. & Avg W.I.P. & Avg Time & Sigma Ratin \\
\hline SEEDS & 1082 & 0 & 0 & 78 & 0 & 1004 & 934.64 & 122315.82 & 6.00 \\
\hline OIL & 78 & 78 & 0 & 0 & 0 & 0 & 0.26 & 480.00 & 6.00 \\
\hline
\end{tabular}

Figure 4: throughput

The total process run time was given above as one hundred and forty-one thousand six hundred seconds. The process line warmed up for a total of six thousand seconds. The throughput of a process line is calculated by dividing the total process time by the bottleneck, which in this case is the crusher, which takes about one thousand eight hundred seconds as shown below:

Throughput $=$ total process time $/$ Bottleneck

$=141600 / 1800$

$=78$ units

Therefore, in kilograms would be $7800 \mathrm{Kg}$

Efficiency $=($ output $/$ Input $) \times 100$

$=(78 / 1082) \times 100$

$=7.2 \%$

As we can see from figure 4, the throughput is correct but the amount of seeds in for that throughput is outrageous hence reducing the efficiency of the process to about seven percent. 


\begin{tabular}{|c|c|c|c|c|c|c|c|c|c|c|}
\hline \multicolumn{11}{|l|}{$\Phi$ WITNESS } \\
\hline \multicolumn{11}{|c|}{ Machine Statistics Report by On Shift Time } \\
\hline Name & \% Idle & $\%$ Busy & $\%$ Filling & \% Emptying & $\%$ Blocked & $\%$ Cycle Wai & $\%$ Setup & \% Setup Wa & \% Broken D & \% Repair W \\
\hline FILTER & 66.95 & 33.05 & 0.00 & 0.00 & 0.00 & 0.00 & 0.00 & 0.00 & 0.00 & 0.00 \\
\hline DRYER & 0.00 & 6.61 & 0.00 & 0.00 & 93.39 & 0.00 & 0.00 & 0.00 & 0.00 & 0.00 \\
\hline HOPPER & 0.00 & 18.73 & 0.00 & 0.00 & 81.27 & 0.00 & 0.00 & 0.00 & 0.00 & 0.00 \\
\hline CRUSHER(1) & 0.00 & 100.00 & 0.00 & 0.00 & 0.00 & 0.00 & 0.00 & 0.00 & 0.00 & 0.00 \\
\hline CRUSHER(2) & 0.00 & 100.00 & 0.00 & 0.00 & 0.00 & 0.00 & 0.00 & 0.00 & 0.00 & 0.00 \\
\hline \begin{tabular}{|l|} 
BAGGING \\
\end{tabular} & 93.64 & 6.36 & 0.00 & 0.00 & 0.00 & 0.00 & 0.00 & 0.00 & 0.00 & 0.00 \\
\hline BOTTLING & 89.83 & 10.17 & 0.00 & 0.00 & 0.00 & 0.00 & 0.00 & 0.00 & 0.00 & 0.00 \\
\hline BAG_SEALE & 98.73 & 1.27 & 0.00 & 0.00 & 0.00 & 0.00 & 0.00 & 0.00 & 0.00 & 0.00 \\
\hline BOTTLE_SE & 97.97 & 2.03 & 0.00 & 0.00 & 0.00 & 0.00 & 0.00 & 0.00 & 0.00 & 0.00 \\
\hline
\end{tabular}

Figure 5: machine usage

The machine usage from figure 5 is as it expected as the bottleneck of process, which is the crusher are shown be one hundred percent busy while the bagging and bottling process are not that busy due to the length of time of crushing. We notice form the figures that since more oil is produced that cake, the bottle sealer is busier than the bag sealer.

\subsection{PROCESS OPTIMIZATION}

To optimize this process, the throughput has been calculated, the efficiency of the process line also calculated and then the results were compared to the simulation and hence the following observations were made. The present model has a buffer, which means more seeds are let into the process and an unequal amount are let out. The crusher is the bottleneck, which is the longest process in the chain but from research with the application of heat as long as it is less that ninety degrees, the time it takes to crush the seeds will be far less and where this is not possible, the number of crushers will be increased therefore speeding up the process time and form experimentations when the buffer is taken out the amount of product is much more closer in number to the amount of raw materials going into the system.

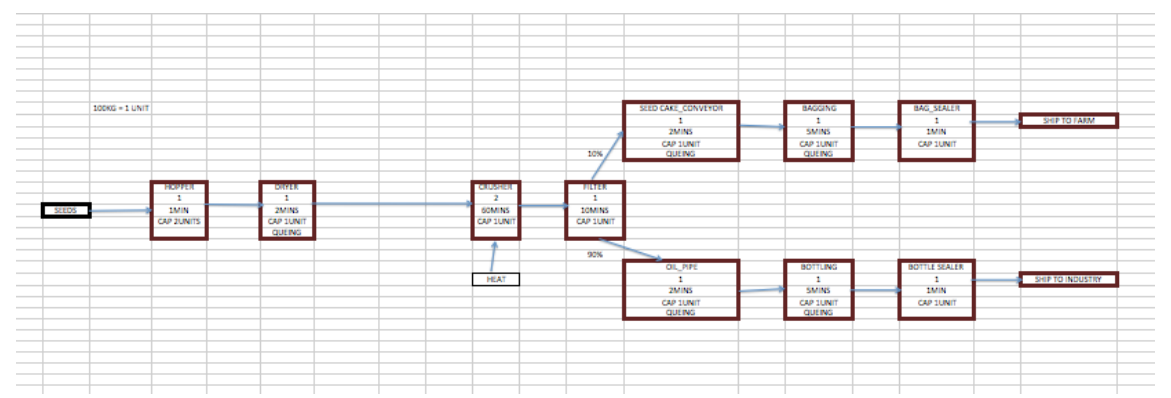

Figure 6: optimized process flow chart

The flow chart above shows the buffer has been removed; heat of no more than ninety degrees is now being supplied to the crusher to ease crushing and cake passage. 


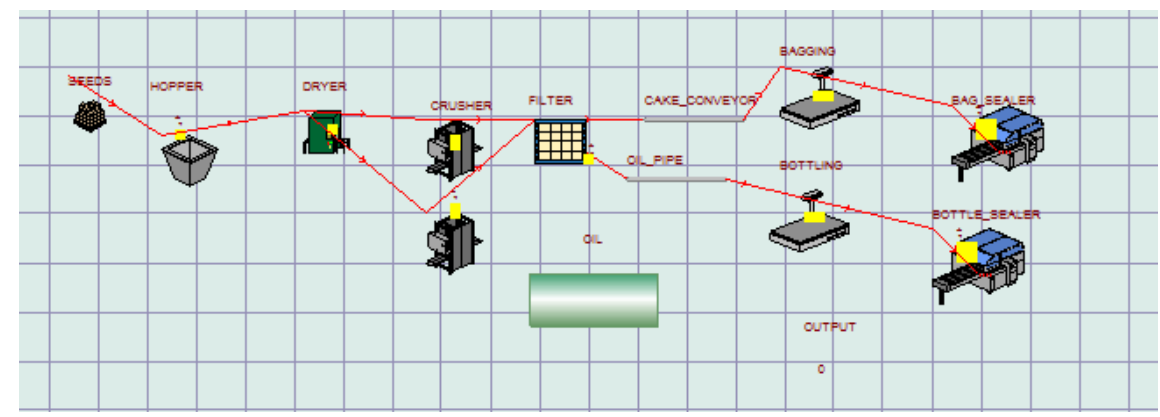

Figure 7: witness simulation

The figure above shows the witness model of the process line as it has been optimized and in the figure below the output of the optimized model process line is shown. The realistic output is seventy-eight, but the model always shows an output which is one less than the realistic.

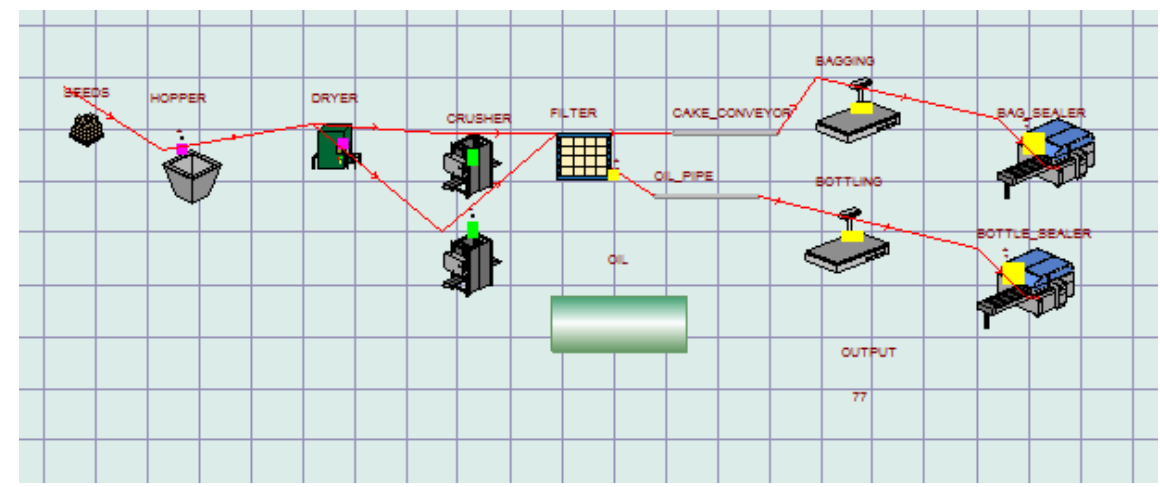

Figure 8: optimized output

\begin{tabular}{|c|c|c|c|c|c|c|c|c|c|}
\hline \multicolumn{10}{|c|}{ Part Statistics Report by On Shift Time } \\
\hline Name & No. Entered & No. Shippe & No. Scrapp & No. Assem & No. Rejecte & W.I.P. & Avg W.I.P. & Avg Time & Sigma Ratin \\
\hline SEEDS & \begin{tabular}{|r|}
82 \\
\end{tabular} & 0 & 0 & 78 & 0 & 4 & 4.50 & 7763.41 & 6.00 \\
\hline OIL & 78 & 78 & 0 & 0 & 0 & 0 & 0.26 & 480.00 & 6.00 \\
\hline
\end{tabular}

Figure 9: optimized throughput

The total process run time used for the optimized process was as given above as one hundred and fortyone thousand six hundred seconds. The process line warmed up for a total of six thousand seconds. The throughput of the process line is once again calculated by dividing the total process time by the bottleneck, which in this case is the crusher, which takes about one thousand eight hundred seconds as shown below:

Throughput $=$ total process time $/$ Bottleneck $=141600 / 1800=78$ units

Therefore, in kilograms would be $7800 \mathrm{Kg}$

Efficiency $=$ (output / Input) $\times 100$

$=(78 / 82) \times 100$

$=95 \%$ 
It is evident from figure 9, the throughput is correct and also matches the calculated value. The amount of raw materials in which in this case are seeds is also commensurate to the amount out and this automatically improves the efficiency to about ninety five percent. The optimized process also means that the product from the filter which is divided using the percentage rule according to the efficiency now transfers ninety five percent of the filtered produced which is oil to the oil pipe while the remaining five percent which is seed cake is taken to the cake conveyor.

\begin{tabular}{|c|c|c|c|c|c|c|c|c|c|c|}
\hline \multirow{2}{*}{\multicolumn{11}{|c|}{ Machine Statistics Report by On Shift Time }} \\
\hline & & & & & & & & & & \\
\hline Name & \% Idle & \% Busy & $\%$ Filling & \% Emptying & $\%$ Blocked & $\%$ Cycle Wail & $\%$ Setup & \% Setup Wa & \% Broken D & $\%$ Repair W \\
\hline FILTER & 66.95 & 33.05 & 0.00 & 0.00 & 0.00 & 0.00 & 0.00 & 0.00 & 0.00 & 0.00 \\
\hline DRYER & 0.00 & 6.61 & 0.00 & 0.00 & 93.39 & 0.00 & 0.00 & 0.00 & 0.00 & 0.00 \\
\hline HOPPER & 0.00 & 1.65 & 0.00 & 0.00 & 98.35 & 0.00 & 0.00 & 0.00 & 0.00 & 0.00 \\
\hline CRUSHER(1) & 0.00 & 100.00 & 0.00 & 0.00 & 0.00 & 0.00 & 0.00 & 0.00 & 0.00 & 0.00 \\
\hline CRUSHER(2) & 0.00 & 100.00 & 0.00 & 0.00 & 0.00 & 0.00 & 0.00 & 0.00 & 0.00 & 0.00 \\
\hline \begin{tabular}{|l|} 
BAGGING \\
\end{tabular} & 99.58 & 0.42 & 0.00 & 0.00 & 0.00 & 0.00 & 0.00 & 0.00 & 0.00 & 0.00 \\
\hline BOTTLING & 83.90 & 16.10 & 0.00 & 0.00 & 0.00 & 0.00 & 0.00 & 0.00 & 0.00 & 0.00 \\
\hline BAG_SEALE & 99.92 & 0.08 & 0.00 & 0.00 & 0.00 & 0.00 & 0.00 & 0.00 & 0.00 & 0.00 \\
\hline BOTLEE_SE & 96.78 & 3.22 & 0.00 & 0.00 & 0.00 & 0.00 & 0.00 & 0.00 & 0.00 & 0.00 \\
\hline
\end{tabular}

Figure 10: optimized machine usage

The machine usage from figure 10 is still as is expected. The bottleneck of process, which is the crusher are shown to still be one hundred percent busy while as compared to the previous operation, the bottling and bottle sealing process is busier than the bagging and bag sealing process.

Figure eleven below shows another modification that can be made to the process line in order to improve its operation. The numbers of crushers were increased to four and it was seen that the total amount of oil generated automatically shot up and took lesser time to produce. Although this is an expensive method as in the short run immediate cost of purchasing the machines would be high but in the long run the profit would be way more than the money invested.

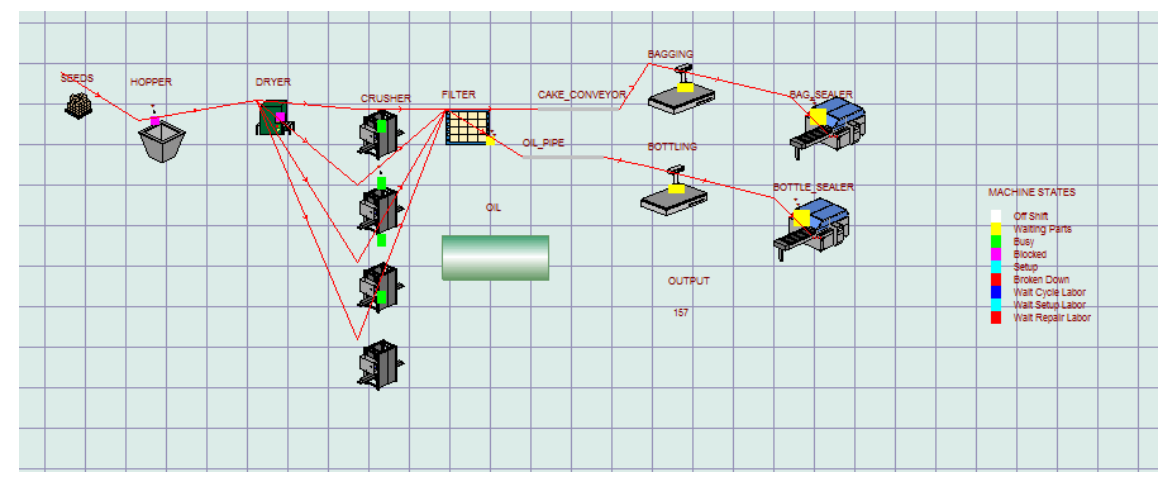

Figure 11: increased crushers

Figure twelve shows the machine statistics of the process line when it is idle. As can be seen from the figure, the yellow square signifies that the machine is awaiting parts or raw materials depending on its location in the process line. All machines are awaiting raw materials in the figure below. 


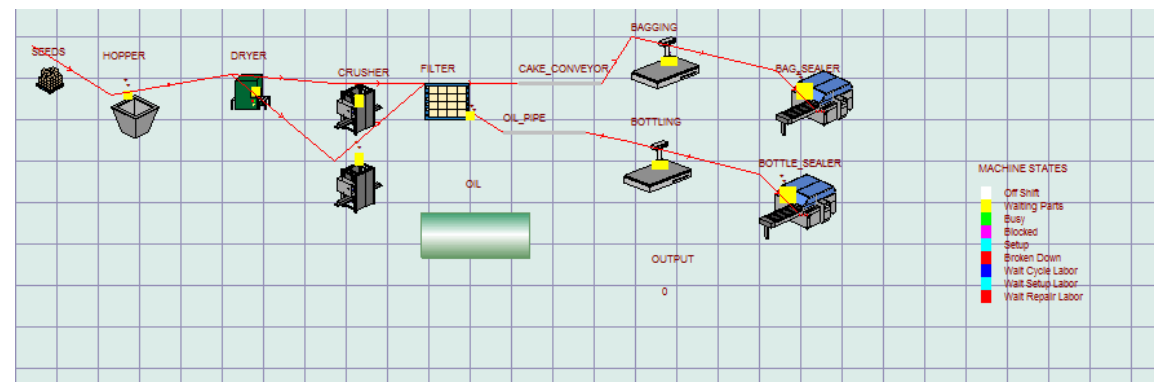

Figure 12: idle system

Figure thirteen shows the machine statistics of the process line when it has undergone its first run. As can be seen from the figure, the yellow square signifies that the machine is awaiting parts or raw materials depending on its location in the process line while the pink square signifies that the machines that are blocked and the green square tells us the machines that are busy. The machines that are awaiting raw materials in the figure below are the filter, the bagging and bottling machines, the bag sealer and bottle sealer machines. The hopper and dryer are blocked but the crushers are busy as expected.

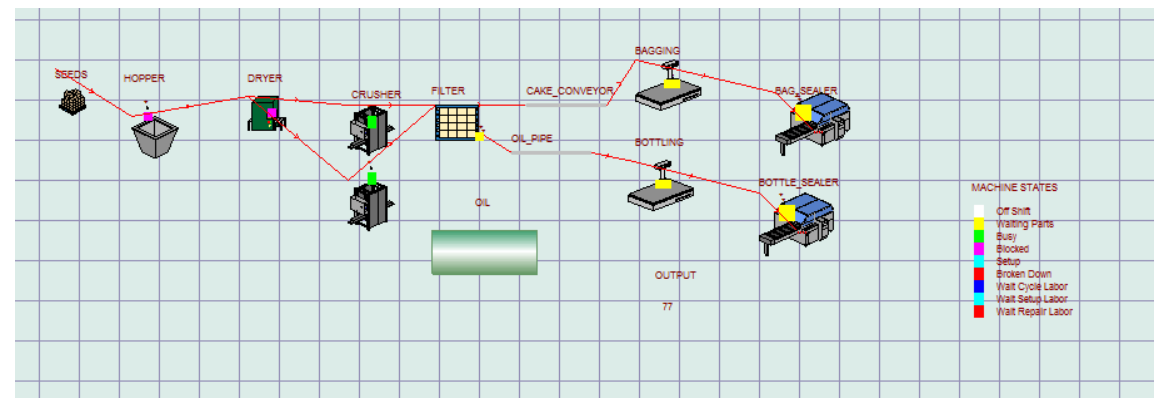

Figure 13: busy system

\subsection{BREAKDOWN}

This is a situation in which any of the machines are not functioning as they should or are not functioning at all. If the breakdown occurs when the machine is in use that is when it is tagged with a green square or idle which would be a yellow square, the breakdown would be known as an available time breakdown. If the machine breakdown is due to overuse, then it is a busy time breakdown while if breakdown occurs due to an unusual amount of operation being carried out with the machine then it is a number of operations breakdowns. This process is liable to all this type of breakdown and the warm up time, which is about six thousand seconds can also be used to cover the repairs of machines involved meanwhile an alternative process line of a backup process line is available and would be switched to in the interim

Labour

Labour is the workforce used to run a process but since this is a completely automated process, there is no need for labour.

\section{Shifts}

Shifts are necessary for workforce or labour hence if there is no labour there is no need for shifts.

\subsection{CONCLUSION}


The use of witness to model the Jatropha oil process line was tasking, as the modelled results were not initially meeting the expected throughput but after careful study and understanding of the process and problem it was found that the buffer was accumulating a whole lot of seeds which was not commensurate to the amount of oil produced but this was found to also be because the buffer capacity was set to one thousand which is way more than we needed. Then it was also discovered that the crusher was accumulating cake hence causing blockage and applying heat not greater than ninety degrees to the process solved this problem. Overall the process was optimized and after being run for a total of about one hundred and fortyone thousand six hundred seconds gave adequate throughput of seventy-eight units of oil which is about seven thousand eight hundred in kilograms as calculated and was realistic.

\subsection{REFERENCES}

1. Chen, H., Defee, C., Gibson, B. and Hanna, J. (2019). Supply Chain Concepts | Defining the Supply Chain | InformIT. [online] Informit.com. Available at: http://www.informit.com/articles/article.aspx?p=2166717\&seqNum=2 [Accessed 12 May 2019].

2. LIQUID DYNAMICS Oil Extractors. 2013. [ONLINE] Available at:http://www.liquidynamics.com/ownerManuals/Oil_Extractors_Manual.pdf. [Accessed 22 January 2013].

3. Machine Design - R.S. Khurmi, J.K. Gupta, Chapter-31. 2013. [ONLINE] Available at:http://www.scribd.com/doc/13657878/Machine-Design-RS-Khurmi-JK-Gupta-Chapter31. [Accessed 22 January 2013].

4. MACHINE DESIGN - The Journal of Faculty of Technical Sciences. [ONLINE] Available at: http://www.mdesign.ftn.uns.ac.rs/. [Accessed 05 October 2012].

5. MECHANICAL HOPPER FEEDER. 2013 [ONLINE] Available at: http://www.freepatentsonline.com/3777908.html. [Accessed 22 January 2013].

6. Onimowo, D. 2013. Modelling and simulation for Operations management.

7. Openshaw, Keith. 2000. A review of Jatropha curcas: an oil plant of unfulfilled promise. Biomass and Bioenergy 19:1-15 